\title{
NOTE ON RELATIVE HOMOLOGICAL DIMENSION
}

\author{
G. HOCHSCHILD*
}

Let $R$ be a ring with identity element 1 , and let $S$ be a subring of $R$ containing 1 . We consider $R$-modules on which 1 acts as the identity map, and we shall simultaneously regard such $R$-modules as $S$-modules in the natural way. In [4], we have defined the relative analogues $\operatorname{Ext}_{(R, S)}^{n}$ of the functors $\operatorname{Ext}_{R}^{n}$ of Cartan-Eilenberg [1], and we have briefly treated the corresponding relative analogues of module dimension and global ring dimension. If $M$ is an $R$-module the relative projective dimension of $M$ is denoted $d_{R, S}(M)$. It is the smallest non-negative integer $n$ (or $\infty)$ for which there is an $R$-module $N$ such that $\operatorname{Ext}_{(R, S)}^{n}(M, N) \neq(0)$. The relative global dimension $\left.d^{\prime} R, S\right)$ of the pair $(R, S)$ is defined as $\sup _{u}\left(d_{R, S}(M)\right)$. We use the similar notations $d_{R}(M)$ and $d(R)$ for the absolute projective $R$-module dimension of $M$ and the global dimension of $R$, respectively.

Our purpose here is to establish some elementary relations between the relative dimensions and the absolute dimensions, and to point out how the relative dimensions can be used to obtain information on absolute dimensions. In particular, we are thereby led to a simple derivation of the known results on the dimension of polynomial rings. In this connection, I have had a number of clarifying discussions with Maurice Auslander, and I wish to thank him here for his valuable comments.

\$1. Following Cartan-Eilenberg, we shall say that $R$ is right $S$-flat if $\operatorname{Tor}_{s}^{n}(R, C)=(0)$, for every $S$-module $C$ and all $n>0$, or, equivalently, if, for every monomorphism $U \rightarrow V$ of $S$-modules, the induced homomorphism $R \otimes{ }_{S} U$ $\rightarrow R \otimes{ }_{S} V$ is a monomorphism. In particular, $R$ is right $S$-flat whenever it is $S$-projective as a right $S$-module.

THEOREM 1. Suppose that $R$ is right $S$-flat. Then, for every R-module $M$,

$$
d_{R}(M) \leqq d_{R, s}(M)+d(S) .
$$

Received June 19, 1957.

* John Simon Guggenheim Fellow. 
If, furthermore, $R$ is S-projective as a left S-module, then

$$
d_{S}(M) \leqq d_{R}(M) \leqq d_{R}, s(M)+d_{s}(M) .
$$

Proof. Since $R$ is right $S$-flat, it is clear that, for every $S$-projective resolution $\ldots \rightarrow X_{1} \rightarrow X_{0} \rightarrow M \rightarrow(0)$ of $M$, the sequence $\ldots \rightarrow R \otimes{ }_{s} X_{1} \rightarrow R \otimes{ }_{s} X_{0}$ $\rightarrow R \otimes{ }_{s} M \rightarrow(0)$ is an $R$-projective resolution of $R \otimes_{s} M$. Hence $d_{R}\left(R \otimes \otimes_{s} M\right)$ $\leqq d_{s}(M)$.

In proving the inequalities involving $d_{R}, s(M)$, we may evidently assume that $d_{R, s}(M)$ is finite, and we shall proceed by induction on $d_{R, s}(M)$. Suppose first that $a_{R, s}(M)=0$. This means that $M$ is $(R, S)$-projective, and hence a direct $R$-module summand of $R \otimes{ }_{S} M$ (see $\$ \S 1,2$ of [4]). Hence $d_{R}(M)$ $\leqq d_{R}\left(R \otimes{ }_{S} M\right) \leqq d_{S}(M)$.

Now assume that $d_{R, s}(M)>0$, and consider the standard $(R, S)$-exact sequence

$$
(0) \rightarrow K \rightarrow R \otimes)_{S} M \rightarrow M \rightarrow(0) .
$$

Since $R \otimes \otimes_{s} M$ is $(R, S)$-projective, we have $d_{R}, s(K)=d_{R, s}(M)-1$. Also, for every $n>0$ and every $R$-module $C$, we have the exact sequence

$$
\operatorname{Ext}_{R}^{n-1}\left(R \otimes{ }_{s} M, C\right) \rightarrow \operatorname{Ext}_{R}^{n-1}(K, C) \rightarrow \operatorname{Ext}_{R}^{n}(M, C) \rightarrow \operatorname{Ext}_{R}^{n}\left(R \otimes{ }_{S} M, C\right) .
$$

We take $n=d_{R, s}(M)+d(S)+1$, or $n=d_{R, s}(M)+d_{s}(M)+1$, according to whether we deal with the first part of Theorem 1, or with the second part. Since $d_{R}\left(R \otimes{ }_{S} M\right) \leqq d_{S}(M)<n-1$, the groups at the ends of our sequence are (0). Hence

$$
\operatorname{Ext}_{R}^{n-1}(K, C) \approx \operatorname{Ext}_{R}^{n}(M, C) .
$$

Now we prove the first part of Theorem 1: here we take $n=d_{R, s}(M)$ $+d(S)+1$, and the inductive hypothesis gives $d_{R}(K) \leqq d_{R, s}(M)-1+d(S)$ $=n-2$. Hence we conclude that $\operatorname{Ext}_{R}^{n}(M, C)=(0)$, whence $d_{R}(M)<n$, i.e., $d_{R}(M) \leqq d_{R, s}(M)+d(S)$.

Now we assume that $R$ is $S$-projective as a left $S$-module and prove the second part of Theorem 1: note first that our assumption implies that every $R$-projective resolution is also an $S$-projective resolution, whence $d_{S}(M) \leqq d_{R}(M)$. Also, $d_{s}(R \otimes s M) \leqq d_{R}\left(R \otimes{ }_{s} M\right) \leqq d_{s}(M)$. Since $K$ is a direct $S$-module summand of $R \otimes s_{S} M$, it follows that $d_{s}(K) \leqq d_{s}(M)$. Hence our inductive hypothesis gives $d_{R}(K) \leqq d_{R, s}(M)-1+d_{s}(M)=n-2$. As above, we conclude that $\operatorname{Ext}_{k}^{n}(M, C)=(0)$, whence $d_{k}(M)<n$, i.e., $d_{R}(M) \leqq d_{R, s}(M)+d_{s}(M)$. 
This completes the proof of Theorem 1.

Note. For the first part of Theorem 1, it is useful to observe that it holds more generally also in the case where, instead of $S \subset R$, we have a given unitary homomorphism $\varphi: S \rightarrow R$, and $R$-modules are regarded as $S$-modules via $\varphi$; the same proof gives $d_{R}(M) \leqq d_{R, \vartheta(S)}(M)+d(S)$, if $R$ is right $S$-flat.

§. We shall use a generalization of a complex defined by Koszul in order to prove the following result on the relative dimension of a polynomial ring.

THEOREM 2. Let $S$ be an arbitrary ring with identity element, and let $R=S\left[x_{1}, \ldots, x_{n}\right]$ be the polynomial ring in $n$ variables $x_{i}$ over $S$. Then $d(R, S)=n$. Moreover, if $M$ is any non-zero R-module that is annihilated by the $x_{i}^{\prime}$ 's, then $d_{R, s}(M)=n$.

Proof. Let $E_{0}=Z$ be the ring of the rational integers, and let $E_{1}$ be the free $Z$-module $Z y_{1}+\ldots+Z y_{n}$ of rank $n$ over $Z$, with generators $y_{i}$. Generally, let $E_{k}(k=0,1, \ldots)$ denote the homogeneous component of degree $k$ of the exterior $Z$-algebra built over $E_{1}$. Let $M$ be any $R$-module. We let $E_{1}$ operate on $R \otimes{ }_{s} M$ such that, for $r \in R$ and $m \in M, y_{i} \cdot(r \otimes m)=x_{i} r \otimes m-r \otimes x_{i} \cdot m$. It is immediately verified that any two $E_{1}$-operations on $R \otimes{ }_{s} M$ commute.

Now set $X_{0}=R \otimes{ }_{s} M$, and let $d_{0}$ be the canonical epimorphism $R \otimes{ }_{s} M \rightarrow M$. Generally, set $X_{k}=R \otimes{ }_{s} M \otimes{ }_{2} E_{k}$, and define the $R$-homomorphism $d_{k}: X_{k} \rightarrow X_{k-1}$ such that, for $u_{i} \in E_{1}$ and $v \in R \otimes \otimes_{s} M$,

$$
d_{k}\left(v \otimes u_{1} \ldots u_{k}\right)=\sum_{i=1}^{k}(-1)^{i-1}\left(u_{i} \cdot v\right) \otimes u_{1} \ldots u_{i-1} u_{i+1} \ldots u_{k} .
$$

Since the $E_{1}$-operators on $R \otimes s M$ commute, it follows as usual that $d_{k-1} \circ d_{k}=0$, so that we have an $R$-complex $(X, d)$ over $M$. Clearly, each $X_{k}$ is $(R, S)$ projective for its natural structure as an $R$-module, and $X_{k}=(0)$ for $k>n$.

Next, we shall exhibit an $S$-homotopy $h$ of the complex $(X, d)$, whence we shall conclude that $(X, d)$ is an $(R, S)$-projective resolution of $M$. We define $h_{-1}: M \rightarrow R \otimes s M=X_{0}$ by $: h_{-1}(m)=1 \otimes m$. Then $h_{-1}$ is clearly an S-homomorphism, and $d_{0} \circ h_{-1}$ is the identity map on $M$. Now we define an S-homomorphism $h_{0}: X_{0} \rightarrow X_{1}$ such that $h_{0}(1 \otimes m)=0$ and, for $q>0$ and $i_{1} \leqq \ldots \leqq i_{q}$,

$$
h_{0}\left(x_{i_{1}} \ldots x_{i_{y}} \otimes m\right)=\sum_{p=0}^{q-1} x_{i_{1}} \ldots x_{i_{p}} \otimes x_{i_{p+2}} \ldots x_{i_{\Psi}} \cdot m \otimes y_{i_{2,1}} \text {. }
$$

Generally, for $k>0$, we define an $S$-homomorphism $h_{k}: X_{k} \rightarrow X_{k+1}$ such 
that, with $1 \leqq j_{1}<\ldots<j_{k} \leqq n$,

$$
\begin{aligned}
& h_{k}\left(1 \otimes m \otimes y_{j_{1}} \ldots y_{j_{k}}\right)=0, \quad \text { and } \quad h_{k}\left(x_{i_{1}} \ldots x_{i_{l}} \otimes m \otimes y_{j_{1}} \ldots y_{j_{k}}\right) \\
& =(-1)^{k} \sum_{r: i_{r+1}>j_{k}} x_{i_{1}} \ldots x_{i_{r}} \otimes x_{i_{r+2}} \ldots x_{i_{q}} \cdot m \otimes y_{j_{1}} \ldots y_{j_{k}} y_{i_{r^{\prime}+1}} .
\end{aligned}
$$

Thus, for instance,

$$
h_{1}\left(x_{1} x_{2} x_{3} \otimes m \otimes y_{1}\right)=-x_{1} \otimes x_{3} \cdot m \otimes y_{1} y_{2}-x_{1} x_{2} \otimes m \otimes y_{1} y_{3} .
$$

One can verify by induction on $q$ that $d_{k+1} \circ h_{k}+h_{k-1} \circ d_{k}$ sends each $x_{i_{1}} \ldots x_{i_{g}} \otimes m \otimes y_{j_{1}} \ldots y_{j_{k}}$ and each $1 \otimes m \otimes y_{j_{1}} \ldots y_{j_{k}}$ onto itself. In doing this, it is convenient to treat separately the case where $i_{q} \leqq j_{k}$ and the case where $i_{q}>j_{k}$. The verification is tedious but presents no essential difficulties; we shall omit it. Once this verification has been made, one concludes that $h \circ d$ $+d \circ h$ is the identity map on $X$, so that $h$ is an $S$-homotopy.

Since $X_{k}=(0)$ for $k>n$, it follows that $d(R, S) \leqq n$. Now let $M$ be a non-zero $R$-module that is annihilated by the $x_{i}$ 's. Then we shall see that $\operatorname{Ext}_{(R, S)}^{n}(M, M) \approx \operatorname{Hom}_{s}(M, M) \neq(0)$. Indeed, $\operatorname{Ext}_{(R, S)}^{n}(M, M)$ is the $n$-th cohomology group of the complex whose component of degree $k$ is $\operatorname{Hom}_{R}\left(X_{k}, M\right)$. Consider the coboundary map $\delta_{k}: \operatorname{Hom}_{R}\left(X_{k}, M\right) \rightarrow \operatorname{Hom}_{R}\left(X_{k+1}, M\right)$ of our complex. If $f \in \operatorname{Hom}_{R}\left(X_{k}, M\right)$ we have $\delta_{k}(f)=f \circ d_{k+1}$. Since $x_{i} \cdot M=(0)$, for each $i$, it is seen at once from the definition of $d_{k+1}$ that $d_{k+1}\left(X_{k+1}\right)$ $\subset \sum_{i} x_{i} \cdot X_{k}$. Using again that the $x_{i}$ 's annihilate $M$, and that $f \in \operatorname{Hom}_{R}\left(X_{k}, M\right)$, we see that $f \circ d_{k+1}=0$. Thus $\delta_{k}=0$, whence $\operatorname{Ext}_{(R, s)}^{n}(M, M) \approx \operatorname{Hom}_{R}\left(X_{n}, M\right)$. Since $E_{n}$ is of rank 1 over $Z$, we have $X_{n} \approx R \otimes \otimes_{S} M$, so that $\operatorname{Hom}_{R}\left(X_{n}, M\right)$ $\approx \operatorname{Hom}_{R}\left(R \otimes{ }_{S} M, M\right) \approx \operatorname{Hom}_{S}(M, M)$. Hence we conclude, using that $d(R, S)$ $\leqq n$, that $d_{R, s}(M)=n$ and $d(R, S)=n$. This completes the proof of Theorem 2 .

It is worth while to observe that if $S$ is semisimple the notions of $(R, S)$. projective resolution and $R$-projective resolution coincide, so that $d_{R}, s(M)$ $=d_{R}(M)$ and $d(R, S)=d(R)$. Hence, in that case, Theorem 2 immediately gives the results that $d(R)=n$, and $d_{R}(M)=n$ for every non-zero $R$-module that is annihilated by the $x_{i}$ 's.

If we combine Theorem 2 with a rather simple argument we obtain the following complete results, which were first proved by Eilenberg, Rosenberg and Zelinsky, in [2].

Let $S$ be an arbitrary ring with identity element, and let $R$ be the polvnomial ring $S\left[x_{1}, \ldots, x_{n}\right]$. Then, for every $R$-module $M, d_{S}(M) \leqq d_{R}(M) \leqq n+d_{s}(M)$. 
Moreover, if $M$ is annihilated by the $x_{i}$ 's and is not (0), then $d_{f_{i}}(M)=n+d_{S}(M)$. Hence we have $d(R)=n+d(S)$.

Proof. Clearly, the second part of Theorem 1 applies, so that $d_{5}(M)$ $\leqq d_{R}(M) \leqq d_{R, s}(M)+d_{s}(M)$. The first of the results stated above follows immediately from this and Theorem 2.

We shall prove the second result by induction on $n$. Clearly, it is therefore sufficient to show that, with $R=S[x]$ and $x \cdot M=(0), M \neq(0)$, we have $d_{R}(M)$ $\geqslant 1+d_{s}(M)$.

Let $\ldots \rightarrow X_{1} \rightarrow X_{n} \rightarrow M \rightarrow(0)$ be an $S$-projective resolution of $M$. Then the sequence

$$
\ldots \rightarrow R \otimes{ }_{s} X_{1} \rightarrow R \otimes{ }_{s} X_{0} \rightarrow R \otimes{ }_{s} M \rightarrow(0)
$$

is an $R$-projective resolution of $R \otimes, M$. Let $\zeta$ denote the endomorphism of this complex that is obtained by operating on it with the element $x$. Let $C$ be another $R$-module such that $x \cdot C=(0)$, and consider the complex with components $\operatorname{Hom}_{R}\left(R \otimes s X_{k}, C\right)$. It is clear, because $x \cdot C=(0)$, that the endomorphism of this complex that is induced by $\zeta$, namely the map $f \rightarrow f \circ \zeta$, is 0 . Hence the endomorphism $\zeta^{*}$ of $\operatorname{Ext}_{R}^{n}(R \otimes s M, C)$ that corresponds to $\zeta_{-1}: R \otimes x_{s} M$ $\rightarrow R \otimes{ }_{s} M$ is 0 . On the other hand, the sequence

$$
(0) \rightarrow R \otimes{ }_{S} M_{\zeta-1} R \otimes{ }_{S} M \rightarrow M \rightarrow(0)
$$

is exact, because $x \cdot M=(0)$, and it yields the exact sequence



Since $R \otimes{ }_{s} X$ is an $R$-projective resolution of $R \otimes{ }_{s} M$ and $\operatorname{Hom}_{R}\left(R \otimes{ }_{s} X, C\right)$ $\approx \operatorname{Hom}_{s}(X, C)$, we have $\left.\operatorname{Ext}_{S i}^{p}(R \times)_{s} M, C\right) \approx \operatorname{Ext}_{S}^{p}(M, C)$. Using this, and the fact that $\zeta^{*}=0$, we obtain the following exact sequence from the above

$$
(0) \rightarrow \operatorname{Ext}_{s}^{n}(M, C) \rightarrow \operatorname{Ext}_{R}^{n+1}(M, C) \rightarrow \operatorname{Ext}_{s}^{n+1}(M, C) .
$$

Since $d_{s}(M) \leqq d_{l i}(M)$, we may assume that $d_{s}(M)$ is finite. Take $n=d_{s}(M)$. Then the last sequence gives $\operatorname{Ext}_{S}^{n}(M, C) \approx \operatorname{Ext}_{l i}^{n+1}(M, C)$, which shows that $d_{k}(M) \gtrsim 1+n=1+d_{s}(M)$. Thus we may now conclude by induction that, in the general case, $d_{R}(M I)=n+d_{s}(M)$. Since every $S$-module may be regarded as an $R$-module annihilated by the $x_{i}$ 's, our results imply that $d(R)=n+d(S)$. Our proof is now complete.

\$3. As a final illustration, we shall prove a result on the dimensions for matrix rings, the second part of which is due to M. Harada, [3]. 
THEOREM 3. Let $S_{n}$ be the ring of all $n$ by $n$ matrices over $S$. Then $d\left(S_{n}, S\right)$ $=0$, and hence $d_{s_{n}}(M)=d_{\mathrm{c}}(M)$, for every $S_{n}$-module $M$.

Proof. Consider the canonical epimorphism $S_{n} \otimes_{s} M_{\rightarrow} M$. Using the usual matrix units $e_{i j}$, we define a map $\varphi: M \rightarrow S_{n} \otimes_{s} M$ by setting $\varphi(m)=\sum_{i=1}^{n} e_{i 1}$ ( $e_{1 i} \cdot m$, for every $m \in M$. It is easy to verify directly that $\varphi$ is an $S_{n}$-homomorphism, and that $\pi \circ \varphi$ is the identity map on $M$. Hence $M$ is isomorphic with a direct $S_{n}$-module summand of $S_{n} \otimes{ }_{s} M$, whence $M$ is $\left(S_{n}, S\right)$-projective. Since $M$ is an arbitrary $S_{n}$-module, this means that $d\left(S_{n}, S\right)=0$. Now the second part of Theorem 1 applies to the present situation and shows that $d_{S_{n}}(M)=d_{s}(M)$, for every $S_{n}$-module $M$.

\section{REFERENCES}

[1] H. Cartan and S. Eilenberg, Homological Algebra, Princeton, 1956.

[2] S. Eilenberg, A. Rosenberg and D. Zelinsky, On the dimension of modules and algebras (VIII), Nagoya Math. J., Vol. 12 (1957), 71-93.

[3] M. Harada, Note on the dimension of modules and algebras, J. Inst. Polytech. Osaka City Univ., Ser. A. 7 (1956), 87-96.

[4] G. Hochschild, Relative Homological Algebra, Trans. Am. Math. Soc., Vol. 82 (1956), 246-269.

Added in proof. The arguments of $\S 2$ yield also the following result.

Theorem 4. Let $R$ be the ring of polynomials in an arbitrary set $U$ of freely non-commuting variables, with coefficients in a ring $S$ with 1 , the elements of $S$ commuting with those of $U$. Then $d(R, S)=1$, and $d_{S}(M) \leqq d_{R}(M) \leqq 1$ $+d_{\varsigma}(M)$, for every unitary R-module $M . \quad$ If $M$ is not $(0)$ and is annihilated by some $u \in U$ then $d_{R}(M)=1+d_{s}(M)$.

Proof. Let $X_{0}, X_{1}, d_{0}, d_{1}$ be defined as in the proof of Theorem 2 ; in defining $d_{1}$, operate on $R$ by multiplying from the right. Consider the resulting sequence $(0) \rightarrow X_{1} \rightarrow X_{0} \rightarrow M \rightarrow(0)$. Using $h_{-1}$ and $h_{c}$, as defined in the proof of Theorem 2, we verify that this is an $(R, S)$-projective resolution of $M$. Hence $d(R, S) \leqq 1$. If we take $M \neq(0)$ and such that $U \cdot M=(0)$ we see directly that the sequence cannot split as an $R$-module sequence. Hence $d(R, S)$ =1. The inequalities for the dimensions of $M$ now follow from Theorem 1 .

Now suppose that $M$ satisfies the conditions of the last assertion. Since $R$ is free over $S[u]$, every $R$-projective resolution of $M$ is also $S[u]$-projective,

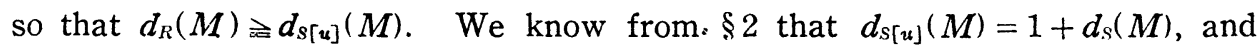
the last assertion of Theorem 4 follows on putting our results together. 